Open Scientist Handbook • OSH

\title{
The Onlyness of the Career Open Scientist
}

Bruce R. Caron

Published on: Mar 09, 2021

DOI: $10.21428 / 8 b b b 7 f 85 . b a 2 d 442 f$

License: Creative Commons Attribution 4.0 International License (CC-BY 4.0). 


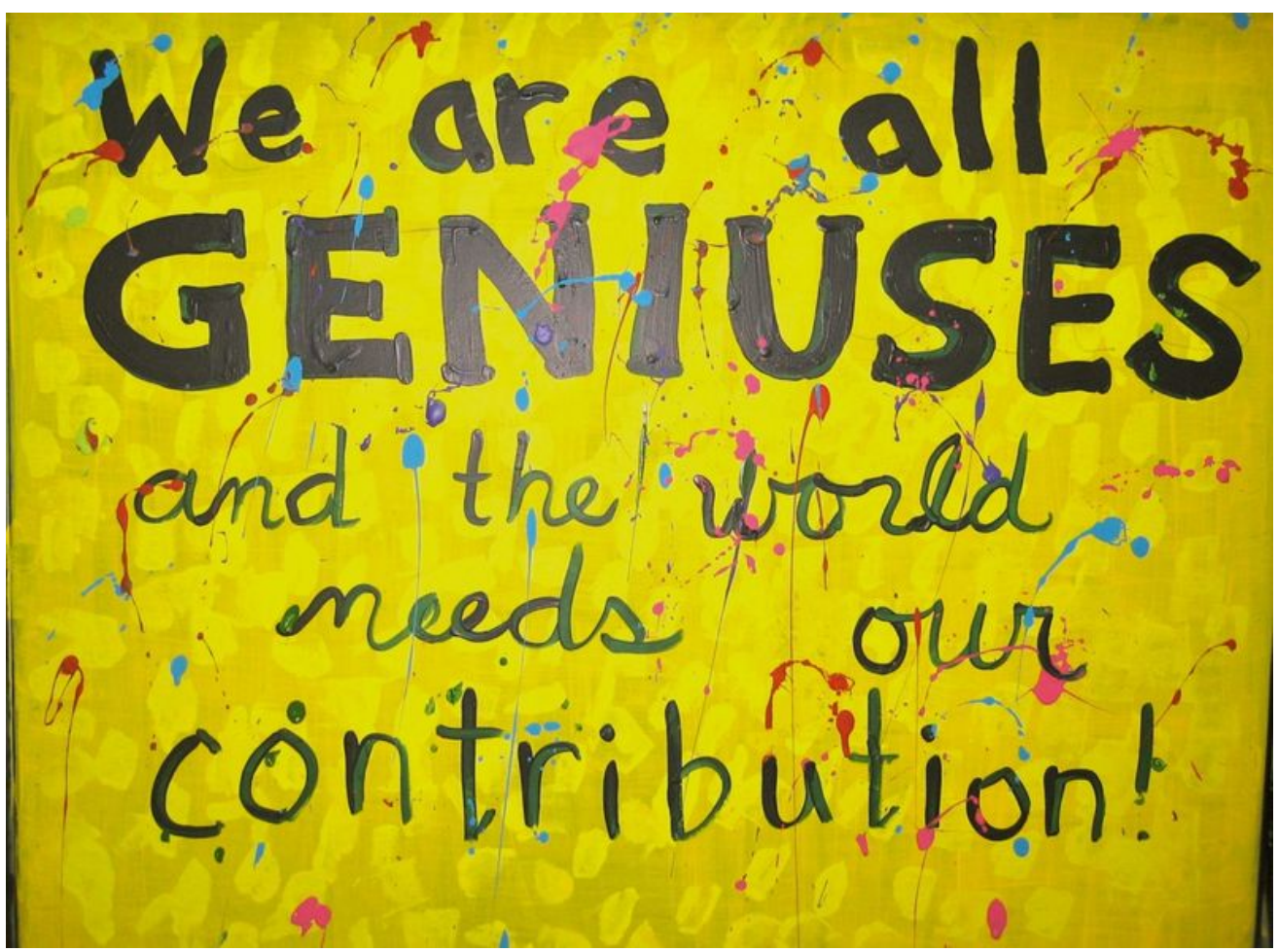

Photo Credit: Denise Krebs on Flickr. CC by 2.0

"Through the power of onlyness, an individual conceives an idea born of his narrative, nurtures it with the help of a community that embraces it, and, through shared action, makes the idea powerful enough to dent the world" (Merchant 2017)

When we look ahead at some near-future, open-science-based academy, we can point to a new science workplace solidly anchored into its own logic, with internal values that reveal its core practices. Each open organization operationalizes these values locally.

Open science is aspirational because science is hopeful: it aspires to add significant new knowledge to human understanding. The open-science movement started with open access to research results. This struggle continues. The current pandemic may accelerate this process. Next, open science needs to move to implement cultural practices that enable Demand Sharing_and Fierce Equality at all levels and organizations. Certain common notions about academy practices-certain notions inherited from decades of science infested by toxic cultural habits and perverse incentives-will need to be interrogated as open science looks to open cultural practices to shape how it changes in the future. Let's examine some of these practices. 


\section{On Onlyness}

But first. The notion of, and the term "onlyness," is from Nilofer Merchant* (2017), an entrepreneur/business consultant (See also: <https://nilofermerchant.com/>; Accessed May 12, 2020). Merchant penned this term to highlight how the quality of ideas in any group springs from the distinct contribution that each person can bring into conversation, when they speak from the totality of their own knowing and being (See: Knowing and conversation). She then outlines how to optimize for this potential. Most teams and organizations downplay, or worse, prohibit, this potential, asking team members to be flexible generalists and leave their individual genius at home. They ask their members/employees to "fit in" instead inviting them to belong on their own terms.

By locating "diversity" and "inclusion" in the distinct features of each individual, Merchant re-places the standard arguments for these values within teams and organizations. No tokenism permitted here. Instead, we find a keen respect for the distinct biographies of learning and knowing carried by each person in their whole being. As Merchant (2017) notes; "To claim yourself as whole is to assert your own value-not because everything about you is perfect but because it is all perfectly yours. This acceptance of your full self is nonnegotiable in claiming the power of onlyness. If you can't value what you alone have to offer, no one else can either."

Onlyness belongs to every individual to the extent they make a claim about it. It is not exclusive or elite; not just a property of egoistic narcissists. It does not automatically lead to assholish behaviors. It is not acquired from membership in a population cohort or a generation, even though it is socially attuned. It is informed throughout your biography, and includes what you alone can bring to your family, your society, and the planet. Einstein's housekeeper had as much onlyness as did Einstein, only Einstein managed to explore and mine his to the advantage of his ability for creative insight.

Very much in line with recent theories of organizational knowledge management (See: Demand sharing and the power of pull), onlyness powers innovation and creativity within teams and projects by surfacing insights across vital conversations. This also amplifies the value of networks over hierarchies. Open innovation studies have shown that "marginal individuals" contribute significantly to solving grand challenges online, in part, by reframing the problems: "The ability of marginal individuals, with different perspectives and heuristics, to come up with novel solutions to broadcast problems, indicates that they may be conceiving of a given problem in a different way than the seeker. Thus problems should not be considered as fixed and given but open to redefinition by the solvers themselves" (Jeppesen and Lakhani 2010). Each team 
member has a contribution to make to this conversation. No more deference to the highest paid person in the room. Are you looking for better conversations (of course you are), then help each person in your research network explore and claim their onlyness.

"[T]he new research suggests that our everyday thinking and learning is strikingly continuous with scientific thinking and learning. The pre-schoolers see probabilistic evidence and revise hypotheses, but they don't necessarily know that that is what they are doing-nor indeed do ordinary adults" (Gopnik 2012).

\section{Onlyness in learning}

There is no textbook sufficient to map the individual's journey into science. There is no common, model individual open scientist: no career best practice, no business-school recipe for success can identify which scientist will excel in their research endeavor. There is no "mold" for an open scientist. Like a concert violinist, a scientist must master techniques and become proficient in their practice and precise in their methods. But that's more like saying they've stopped being children and are now adults. Or, in a curious fashion, that they've stopped trying to be so adult, and have opened up their internal child-driven curiosity and wonder. To be an open scientist, as Berger (2014) notes: "We must become, in a word, neotenous (neoteny being a biological term that describes the retention of childlike attributes in adulthood). To do so, we must rediscover the tool that kids use so well in those early years: the question."

Knowing what the best questions are and how these are situated into the landscape of scientific discovery is the admission price to enter the "knowledge club" (Accessed May 18, 2020) of science. The other side of wonder is rigor. Rigor is learned later in school. You cannot have wonder without rigor. The reverse is also true. "[R]igor cannot be sustained without wonder; and without both capacities, creativity-and innovationwill suffer" (Nixon 2020). However, rigor is not what drives the individual capacity for science. It's a launch utility, not a fuel. You can teach methods and you can learn content. That gets you to the door of science. To open it, you need to apply your own brand of onlyness.

"It was opal and this was something I knew, something I could draw a circle around and testify to as being true. While looking at the graph, I thought about how I now knew something for certain that only an hour ago had been an absolute unknown, and I slowly began to appreciate how my life had just changed. 
I was the only person in an infinite exploding universe who knew that this powder was made of opal. In a wide, wide world, full of unimaginable numbers of people, I was-in addition to being small and insufficient-special. I was not only a quirky bundle of genes, but I was also unique existentially, because of the tiny detail that I knew about Creation, because of what I had seen and then understood" (Jahren 2016).

Onlyness here means deep and broad individuality and intellectual curiosity: open scientists are deep into their own specific passion and love for some aspect of science, and their own corner of the unknown in the infinite play of science. Anyone who has completed a dissertation knows the onlyness-and also the loneliness-of understanding something deeper than their books, better than their advisors, and newer than anyone else. Open scientists are also broad enough in their sense of how science works and in the landscape of methods and literature to see the larger picture of open science practice shared in their discipline and beyond.

Onlyness is the reason why scientists can uncover astonishing new insights. By "scientists" here, we mean all students of science, all members of a science team (data nerds, technical specialists, grad students, and principle investigators), in fact, all people on the planet who find that their curiosity-and their own life-to-right-now-has moved them to a distinct point of understanding: "You're standing in a spot in the world that only you stand in, a function of your history and experiences, visions, and hopes. From this spot where only you stand, you offer a distinct point of view, novel insights, and even groundbreaking ideas. Now that you can grow and realize those ideas through the power of networks, you have a new lever to move the world" (Merchant 2017). Onlyness is why your dissertation nearly drove you insane, since it required you to dive deep into a personal journey of discovery that now means you know more-and you know differently-from anyone else on the planet.

If professional scientists act similarly, this similarity comes from the shared depth of their appreciation for the "role of ignorance and the importance of uncertainty" in science (See: Brain Pickings; Accessed May 5, 2020). Rather like we all are in the current situation (now being May of 2020), scientists are, and have always been, alone, even when together.

\section{Onlyness and fierce equality}

Institutional prestige is a profound drag on the potential for networked science. If your administration has a plan to "win" the college ratings game, this plan will only make 
doing science harder. It makes being a scientist less rewarding. Playing finite games of chasing arbitrary metrics or bullshit prestige drags scientists away from the infinite play of actually doing science. In a world where science happens elsewhere, the first thing your campus can do is become more attached to all those academy "elsewheres" that can amplify your in-house efforts.

The best thing your campus can do is to became that really attractive "elsewhere" to which others want to attach themselves. This means opening up to demand sharing. Once science gets funded across a broad spectrum of institutions and across the globe, online collaboratives will form, and work together, and create new knowing without regard to game-able institutional rankings. The entire academy will become more nimble, creativity will quicken, and good work will find its rewards outside of current reputation schemes.

"One will weave the canvas; another will fell a tree by the light of his ax. Yet another will forge nails, and there will be others who observe the stars to learn how to navigate. And yet all will be as one. Building a boat isn't about weaving canvas, forging nails, or reading the sky. It's about giving a shared taste for the sea, by the light of which you will see nothing contradictory but rather a community of love" (Saint-Exupéry 1948; translation: < https://quoteinvestigator.com/2015/08/25/sea/>; Accessed May 11, 2020).

The future of open science will be much more distributed and democratic. Open scientists work wherever their research and teaching acumen is needed and supported. The perverse lure of bullshit-prestige institutions disappears as great work emerges from highly diverse teams in hundreds of institutions and locales across the planet, and along the internet.

Instead of boasting of their employment at some famous university or lab, open scientists and their in-house and online teams are deep into infinite play wherever they are employed. They shape the culture of their teams, bending this toward fierce equality and demand sharing. Their combined onlynesses serves to push the team effort beyond what any one of them might do. They fill open repositories with new data and findings. They care for their work together, and for each other as people. They celebrate their team culture. A great team in a sad organization with a toxic culture works better than a sad team in a great organization. 


\section{Onlyness against neoliberal organizational metrics}

The notion that a university can increase managerial control over research practices using performance-based funding schemes, and so to capture year-by-year productivity gains, has been tried in various places on the globe. But the practice of top-down, goaldriven, productivity management translates poorly from the commercial world (where this is also failing) into the academy. Metrics applied in this manner are highly susceptible to Goodhart's law, and subsequent gaming attempts. The best incentives for better science are those goods internal to the professional practices of doing science. The best way to improve on these is to support governance practices that open up more avenues for sharing and knowing.

There is an authentic "meritocracy," here, not the artificial sort claimed by prestigious organizations. A fluid, dynamic, emergent shared sense of where new knowing is being forged. In the interconnected intellectual rooms of online science communities, the acceleration of knowing and discovery through access to open shared resources, active, global collaborations, and diverse team-building assembles shared intelligence to solve wicked problems. There is no organizational strategic plan, no business model, no tactical hiring that can match open innovation collaboratives that push the boundaries and change the rules of their infinite playing together. The merit belongs to the team, and to the work. What the scientists get is the joy and wonder of a lifetime of science play.

\section{Open science optimizes resources when onlyness gets support across organizations}

Doing open science gets a boost when the culture of open science is shared and celebrated at the top level of academy institutions-whether its a college, a university, a learned society, or a science agency. Under these circumstances, institutional values and their shared meanings cascade down across all departments, labs, and teams. With solid, top-level institutional support, teams build their own shared mini-cultures to encourage caring and rigor.

The cultural project of open science is actually quite small. As much as open science is just "science done right" and a "return to former science norms," the professed culture of most science organizations really only needs to be rearticulated for open science use, and celebrated as an active, reflexive cultural layer (See above). Goals are left to teams to identify and activate for themselves. 
All biographies of "notable scientists" spend a great amount of their content describing the onlyness of these individuals (without calling it that). Every "genius" you read about is someone who managed to tap into their onlyness and find insights into infinite play (which is commonly referred to as a "serendipitous" event). Every one around them-these individuals are nearly always surrounded by colleagues-contributed to these conversations. They need to noted too.

\section{Science organizations create prizes that fail to capture the onlynesses of teams and collaboratives}

"In the end, it cannot be doubted that each of us can see only a part of the picture. The doctor sees one, the patient another, the engineer a third, the economist a fourth, the pearl diver a fifth, the alcoholic a sixth, the cable guy a seventh, the sheep farmer an eighth, the Indian beggar a ninth, the pastor a tenth. Human knowledge is never contained in one person. It grows from the relationships we create between each other and the world, and still it is never complete" (Kalanithi 2016).

No scientist has ever refused a Nobel Prize. (NOTE: Two people have declined this. Jean-Paul Sartre did, because he was Sartre. And, Le Duc Tho did, because his award was shared with Henry Kissinger, who supported bombing Hanoi during Christmas.) Learned societies and professional academic groups offer a wide range of honors that scientists gladly accept (usually along with travel support to a meeting they would like to attend). Other honors come directly from universities or funding agencies. All of these honors fatten résumés and grease promotion portfolios around the planet.

Scientists crave the economic support they need to do the work they love. If they can translate honors into cash, they will do so. And yet, should these particular honors stop being awarded, there is little to indicate that science would be less rewarding or less able to track the value of science work. Open science offers to expand opportunities to find and use great science. New methods of acknowledging the work of scientists and teams, and also the provenance of research can replace or enhance how scientists get connected to each other through their work.

Great work in open science can be found anywhere on the planet, and also within any team working in an open-science organization, small or large. The gifts of new knowledge are freely shared, but they also obligate others to pay attention. applaud their value, and scorn those who seek attention for their own finite game. This is central to the culture of demand sharing. Recognize the work. Applaud the teams, the 
history, and the ideas. Show appreciation for how this knowledge is shared, without needing to pin this discovery on an individual scientist.

"Scientists are like those levers or knobs or those boulders helpfully screwed into a climbing wall. Like the wall is some cemented material made by mixing knowledge, which is a purely human construct, with reality, which we can only access through the filter of our minds. There's an important pursuit of objectivity in science and nature and mathematics, but still the only way up the wall is through the individual people, and they come in specifics-the French guy, the German guy, the American girl. So the climb is personal, a truly human endeavor, and the real expedition pixelates into individuals, not Platonic forms. In the end it's personal, as much as we want to believe it's objective" (Levin 2016).

\section{You can help your students grow their onlyness too}

Growing and tapping into your onlyness is not just a trait that the best open scientists share, it's a trait that open science needs to support, to grow, and to reward. Open science is the common work of millions of un-common individuals. The education of an open scientist requires a healthy dose of infinite-game training, the unleashing of purpose and imagination, and courage and caring in service of collective knowing. In the end, it is the research goal that becomes the teacher, and every scientist plays this alone, even within their team. Each scientist contributes the difference they have cultivated in their own insights to the collective knowing of their team.

To help your students develop their onlyness requires that you promote in them the courage to be essentially different from those around them, and also the honesty and humility to recognize the onlyness of others. Mainly you do this by working on your own onlyness and showing how this matters. You cement your knowledge on the wall of science, daring your students to step up to a new level through you. You share conversations with them that provoke responses only they can provide. Be open to learn from their knowing.

Several authors in the past decades-from Foucault and Illich to Robinson and Godinhave pointed out that factory-style schooling diminishes onlyness (without calling it thus) in favor of standardized understandings and personal disempowerment. Open science will need to also work on the cultures of learning and teaching science. Like practical wisdom (See: The practical wisdom of doing_science), onlyness needs to be exercised for it to grow. 
Merchant's work on onlyness was designed to help companies become more creative and innovative through the collective conversations of their employees, powered by an organizational culture that welcomed each of them to belong to these conversations in their wholeness. What she offers to businesses holds true for the academy, and most particularly in the academy, where onlyness powers great science through networked collaborations.

- Many thanks for Nilofer Merchant's comments on a draft of this section.

\section{Bibliography: Oppen Scientist Handbook References}

